\title{
A STATISTICAL STUDY OF THE RELATIONSHIP BETWEEN X-RAY, OPTICAL AND RADIO LUMINOSITY FOR A SAMPLE OF QSOB
}

\author{
D. M. Worrall ${ }^{1}$, P. Giommi ${ }^{2}$, H. Tananbaum ${ }^{1}$, and G.Zamorani ${ }^{3}$ \\ 1. Harvard-Smithsonian Center for Astrophysics, Cambridge, MA, U.S.A. \\ 2. EXOSAT Observatory, ESA-ESOC, Darmstadt, F.R.G. \\ 3. Istituto di Radioastronomia, Bologna, Italy.
}

ABSTRACT. Our statistical study finds that the $X$-ray luminosity, $l_{x}$, for an average QSO of given optical luminosity, $l_{o}$, is an increasing function of radio luminosity, $l_{r}$. An average QSO of given $l_{o}$ and $l_{r}$ has higher $l_{x}$ if the radio spectrum is flat than if it is steep. The correlation between $l_{x}, l_{o}$ and $l_{r}$ appears to be improved if $l_{r}$ refers only

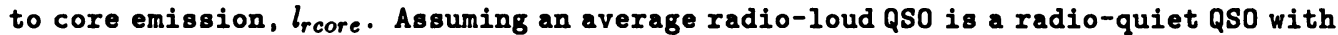
a separate component which produces $l_{\text {rcore }}$ and $l_{x c o r e}, l_{x c o r e} / l_{\text {rcore }}$ is larger if the core is that of a QSO with extended radio structure than if that of a compact flat-spectrum QSO.

Various authors have found that samples of QSOs exhibit a dependence of X-ray luminosity, $l_{x}$, on optical luminosity, $l_{o}$, of the form

$$
\log l_{x}=\log \left(A l_{o}^{\alpha}\right)
$$

(The logarithm of the function is given to illustrate that the regression analysis is for $\log l_{x}$ rather than $l_{x}$ ). Different QSO samples have given values of $\alpha$ between $\approx 0.5$ and $\approx 0.9$ (e.g., Avni and Tananbaum 1986, and references therein). However, it has also been pointed out that the X-ray luminosity of QSOs is influenced by their radio luminosity, $l_{r}$ (e.g., Kembhavi, Feigelson and Singh 1985; and references therein). We have studied this dependence by applying eq. [1] to a sample of $227 \mathrm{QSOs}$ divided into 3 subsamples in bands of $\log \left(l_{r} / l_{o}\right)$. Our fits use the Detections and Bounds method of Avni et al. (1980). Luminosities refer to monochromatic source-frame values at $5 \mathrm{GHz}$, $2500 \mathrm{~A}$ and $2 \mathrm{keV}$ for $l_{r}, l_{o}$ and $l_{x}$, respectively. The $\mathrm{X}$-ray measurements were made with the Einstein Observatory Imaging Proportional Counter. The radio and optical data were taken from many published sources. We find that all 3 subsamples can be fit with the same value of $\alpha$, but the normalization, $A$, increases with increasing $\log \left(l_{r} / l_{o}\right)$. Furthermore, we find that, for the 114 QSOs in our 2 subsamples of highest $\log \left(l_{r} / l_{o}\right)$, i.e., those QSOs we define as radio-loud, $A$ is greater for objects with flat radio spectra (FRS) than for objects with steep radio spectra (SRS).

For investigative purposes, we have chosen a model with a simple dependence of $l_{x}$ on both $l_{o}$ and $l_{r}$,

$$
\log l_{x}=\log \left(K l_{o}^{\alpha} l_{r}^{\beta}\right)
$$


For our FRS QSO sample, we find that the sigma of the Gaussian distribution of the residuals for a fit to eq. [2] is significantly smaller than that for a fit to eq. [1], with $\alpha \approx \beta \approx 0.45$. This suggests a connection between the radio luminosity and at least some of the X-ray luminosity for these objects. FRS QSOs are core compact, and the report of $\approx 75 \%$ VLBI visibility for objects of this type (Zensus, Porcas and Pauliny-Toth 1984) suggests their radio cores are typically $\leq 20$ pc. For our SRS QSO sample, $\alpha \approx 0.45$, whereas $\beta$ is smaller but still greater than zero. We have divided the 46 objects in our SRS sample on the basis of radio morphology. Our SRSCompact sample is populated by 16 objects. These are an interesting group for study because their radio structure is typically complex, with structure on all scales between $\approx 10 \mathrm{pc}$ and $\approx 10 \mathrm{kpc}$ (e.g., Pearson and Readhead 1984). Unfortunately, our 16 objects have an insufficient spread in $l_{r}$ for conclusions to be drawn. Our SRSExtended sample is populated by 24 objects. For these objects, when $l_{r}$ refers only to the luminosity within $\approx 30 \mathrm{kpc}\left(H_{o}=50 \mathrm{~km} \mathrm{~s}^{-1} \mathrm{Mpc}^{-1} ; q_{o}=0\right)$ of the core, $l_{\text {rcore, }}$ the fit to eq. [2] appears to be improved, although statistical uncertainties prevent a strong statement.

In order to investigate physical mechanisms, we have fit the samples to a functional form which separates the dependence on optical and radio luminoisty.

$$
\log l_{x}=\log \left(A l_{o}^{\alpha}+B l_{r}^{\beta}\right)
$$

We find that the term in $l_{o}$ for the FRS sample is consistent with that for the radioquiet sample (for the radio-quiet sample, the term in $l_{r}$ is assumed to be negligible). This implies that we may consider a radio-loud QSO as a radio-quiet QSO with a separate component which produces $l_{\text {rcore }}$ and a contribution to $l_{x}$, which we can call $l_{x c o r e}$. This applies also to the SRS-Extended objects, when $l_{r}$ refers only to $l_{\text {rcore. A }}$ value of $\beta \approx 0.88 \mathrm{fits}$ both the FRS and SRS-Extended (core radio) samples. We then find that the ratio of $B$ for the SRS-Extended (core radio) to FRS samples is $5.5 \pm 3.6$ ( $90 \%$ errors). This means that the ratio $l_{x c o r e} / l_{\text {rcore }}$ is larger if the core is that of a QSO with extended radio structure than if that of a compact flat-spectrum QSO. This could be interpreted as due to relativistic beaming depressing the $X$-ray relative to core-radio luminosity in FRS but not SRS QSOs. This is in qualitative agreement with the prediction of the unified scheme of Orr and Browne (1982), although our results do not imply a sufficiently large effect to be in quantitative agreement.

\section{REFERENCES}

Avni, Y., Soltan, A., Tananbaum, H. and Zamorani, G. 1980, Ap.J., 238, 800.

Avni, Y. and Tananbaum, H. 1986, Ap.J., in press.

Kembhavi, A, Feigelson, E.D. and Singh, K.P. 1985, MNRAS, in press.

Orr, M.J.L. and Browne, I.W.A. 1982, MNRAS, 200, 1067.

Pearson, T.J. and Readhead, A.C.S. 1984, VLBI and Compact Radio Sources, eds. R.Fanti et al. (Reidel), p15.

Zensus, J.A., Porcas, R.W., and Pauliny-Toth, I.I.K. 1984, Astr. Ap., 133, 27. 